\title{
Learning to reflect
}

DOI:

10.1038/nrn2243

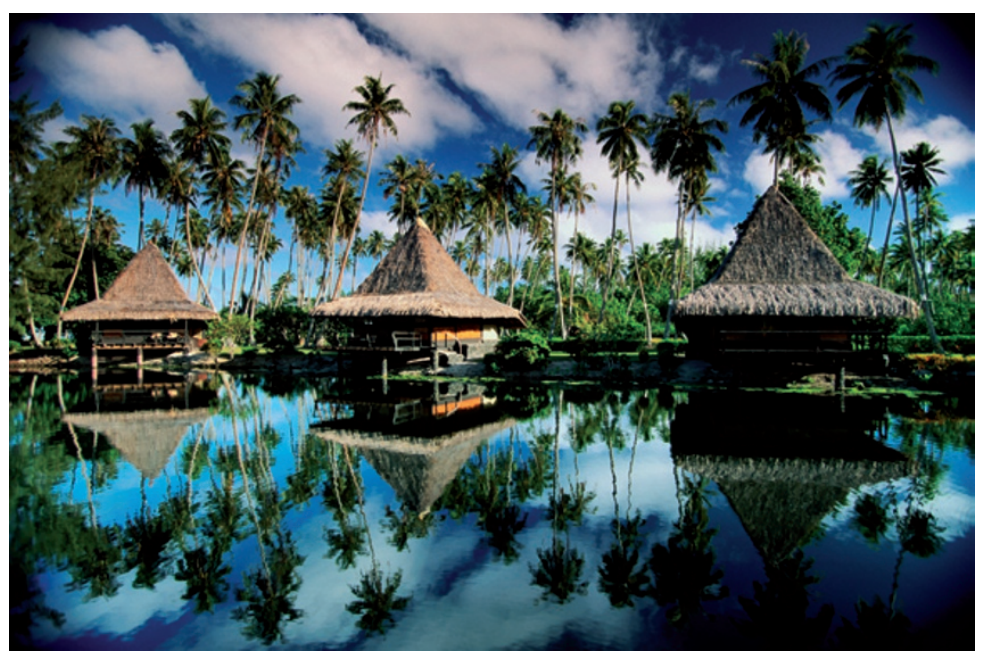

Neurons in the frontoparietal mirror system fire when one performs an action and when one observes someone else performing that same action. This system is thought to have a role in social cognition and, perhaps, in language acquisition. How the mirror neurons map sensory input onto its motor representation is unknown, but Catmur et al. demonstrate that these representations are not innate and can be altered by training.

The authors used transcranial magnetic stimulation (TMS) to stimulate the motor cortex of volunteers who were watching a video of a hand. When the volunteers watched the hand's index finger move, the
TMS-induced motor-evoked potential (MEP) was greater in the abductor muscle of their own index finger than when they watched the little finger move; conversely, the MEP of their little finger's abductor muscle was greatest when they watched the little finger move. In other words, a muscle showed MEP enhancement when its owner watched a movement that is normally performed by that muscle; this 'mirror effect' is thought to reflect activity of the mirror neuron system.

Half of the volunteers then underwent incongruent training trials, in which they were asked to extend their little finger if the video showed a hand extending the index finger, and vice versa. People in congruent trials simply had to repeat the movement they saw in the video. The incongruent trials were assumed to train the mirror system to associate an observed finger movement with movement of a different finger of the volunteer's own hand.

Measuring TMS-induced MEPs after training, the authors found that volunteers who had undergone the incongruent training now showed greater MEPs in the muscle of one finger when watching the 'wrong' finger move in the video, indicating that a reversal of musclespecific MEP enhancement during action observation had taken place.

This study shows that the 'mirror properties' of the mirror system are not innate. Rather, they can be trained, through sensorimotor experience, to transform observation into action. These findings imply that insufficient social interaction and consequent inadequate sensory experience might affect the development of the mirror neuron system, for example, in children with autism. Leonie Welberg

ORIGINAL RESEARCH PAPER Catmur, C. Walsh, V. \& Heyes, C. Sensorimotor learning configures the human mirror system. Curr. Biol. 17, 1527-1531 (2007) 